\title{
AN APPROACH TO SOME NON-CLASSICAL EIGENVALUE PROBLEMS OF STRUCTURAL DYNAMICS
}

\author{
HOREA SANDI - Dr., M., Academy of Technical Sciences of Romania, e-mail: horeasandi@yahoo.com. \\ IOAN SORIN BORCIA ( $\dagger$ ) - Dr., senior researcher, URBAN - INCERC, Romania.
}

\begin{abstract}
Two main shortcomings of common formulations, encountered in the literature concerning the linear problems of structural dynamics are revealed: the implicit, not discussed, postulation, of the use of Kelvin - Voigt constitutive laws (which is often infirmed by experience) and the calculation difficulties involved by the attempts to use other constitutive laws. In order to overcome these two categories of shortcomings, the use of the bilateral Laplace - Carson transformation is adopted. Instead of the dependence on time, $t$, of a certain function $f(t)$, the dependence of its image $f^{\#}(p)$ on the complex parameter $p=\chi+\mathrm{i} \omega(\omega$ : circular frequency) will occur. This leads to the formulation of associated non-classical eigenvalue problems. The basic relations satisfied by the eigenvalues $\lambda_{r}^{\#}(p)$ and the eigenvectors $\boldsymbol{v}_{r}^{\#}(p)$ of dynamic systems are examined (among other, the property of orthogonality of eigenvectors is replaced by the property of pseudo-orthogonality). The case of points $p=p^{\prime}$, where multiple eigenvalues occur and where, as a rule, chains of principal vectors are to be considered, is discussed. An illustrative case, concerning a non-classical eigenvalue problem, is presented. Plots of variation along the $\omega$ axis, for the real and imaginary components of eigenvalues and eigenvectors, are presented. A brief final discussion closes the paper.
\end{abstract}

Keywords: Non-classical eigenvalue problems, Laplace - Carson transformation, pseudoorthogonality, multiple eigenvalues, singular eigenvectors.

\section{Introduction}

The main object of the paper is represented by dealing with non-classical eigenvalue problems encountered in the linear dynamics of structures (having, formally, a finite number, $n$, of degrees of freedom). Matrix formulations are used (vectors: lower case, bold; matrices: upper case bold, characters).

A starting point of further developments is represented by the classical formulation, corresponding to the oscillations of ideally elastic dynamic systems,

$$
M \mathrm{~d}^{2} u / \mathrm{d} t^{2}+K u=f(t)
$$

The matrix $\boldsymbol{M}$ is assumed to be positive definite, while the matrix $\boldsymbol{K}$ is assumed to be positive.

A first further step is represented by the case of systems consisting of materials characterized by Kelvin - Voigt constitutive laws, which leads to the equation of motion

$$
M \mathrm{~d}^{2} u / \mathrm{d} t^{2}+C \mathrm{~d} u / \mathrm{d} t+K u=f(t)
$$

The eigenvalue problem corresponding to the equation of motion (1.1) is defined by the equation

$$
\left(-\mu^{2} \boldsymbol{M}+\boldsymbol{K}\right) \boldsymbol{v}=0
$$

which leads to non-trivial solutions consisting of real eigenvectors $\boldsymbol{v}_{r}$ and eigenvalues $\mu_{r}^{2}(r=1 \ldots n)$.

In case one starts from the equation of motion (1.2), the eigenvalue problem corresponds to the equation:

$$
\left(-\mu^{2} \boldsymbol{M}+\mathrm{i} \mu \boldsymbol{C}+\boldsymbol{K}\right) \boldsymbol{v}=0
$$

(i: imaginary unit) which may lead in general to complex eigenvectors vr and always leads to complex eigenvalues $\mu \mathrm{r}$. It is shown [3], [10] that the eigenvalue problem (1.4) is reducible to a problem (1.3) if and only if the matrix $\mathrm{C}$ may be represented as a linear combination of an 
arbitrary number of terms $[\mathrm{K}(\mathrm{M}(-1) \mathrm{K}) \mathrm{j}]$ (j: real, integer, arbitrary). If so, the eigenvectors $\boldsymbol{v}_{r}$ keep being real, while the eigenvalues $\mu_{r}$ remain real only if the matrix $\boldsymbol{C}$ is equal to zero. The matrix $C$ is non-negative, since in case of existence of damping the process of deformation of the structure is exo-thermal.

Looking at equations (1.2) and (1.4), it turns out that they correspond only to cases when the constitutive laws characterizing the members of the structure are of Kelvin - Voigt type. Otherwise, the analytical expressions (1.2) and (1.4) should change radically, involving considerable additional difficulties for the analysis of dynamic phenomena. An attempt at dealing with various linear constitutive laws, adopted in the paper, consists of using a passage from the use of functions depending on the time variable to that of use of the complex argument $p$. This latter way is subsedquentlyu used.

Starting from these data, the further sections of the paper are concerned with:

- use of Laplace - Carson transforms;

- further discussion on the implications of use of Kelvin - Voigt constitutive laws;

- discussion on the implications determinned by the adoption of the Kelvin - Voigt constitutive law;

- analysis of the dynamic performance of dynamic systems and deriving of specific structural characteristics;

- (in Annex I): additional analysis of specific structural characteristics;

- (in Annex II): additional discussion on alternative cases of applying the dynamic )on.

An illustrative (non-classical case of dealing with dynamic systems is presented.

The paper is concluded by some summary considerations.

\section{Use of the Laplace - Carson transform}

The specific tool for calculations, used in the paper, is represented by the bilateral Laplace Carson transform [9], according to which the relations between the original functions $g(t)$ and their corresponding image functions $g^{\#}(p)$ are:

$$
\begin{array}{ll}
g^{*}(p)=p \int_{-\infty}^{\infty} \mathrm{e}^{-p t} g(t) \mathrm{d} t & \text { where } \chi=\operatorname{Re} p €(\alpha, \beta) \\
\left.g(t)=(1 / 2 \pi \mathrm{i}) \int_{c-\mathrm{i} \infty}^{c-\mathrm{i} \infty}\left[\mathrm{e}^{p t} g^{*}(p) / p\right)\right] \mathrm{d} p & \text { where } c €(\alpha, \beta)
\end{array}
$$

The complex argument used, which replaces for image functions the time variable $t$, on which original functions depend, is denoted $p=\chi+\mathrm{i} \omega$. Its real part, $\chi$, must belong to the convergence band $(\alpha, \beta)$ of the transformation, specific to the original function $g(t)$, in order to make use of the transform referred to. The relations $(2.1)$ can be presented in a shorter conventional version, $\left[g(t) \rightarrow g^{*}(p)\right]$ or $\left[g^{*}(p) \leftarrow g(t)\right]$.

The use of the transform referred to involves the availability of:

- a grammar, which specifies the operations on images corresponding to various operations on originals;

- a dictionary, which specifies the image functions corresponding to various original functions.

The main advantage of using of the Laplace - Carson integral transform is due to the fact that operations to derivate or integrate the original expressions of various functions are replaced by deriving algebraic expressions subjected to multiplication with an integer (positive or negative power) of the variable $p$. The most frequentlly used rules of grammar: determining the derivative with respect to $t$ of the originals, becomes multiplication with $p$ for the images, while simple integration with respect to $t$ of the originals becomes division by $p$ for them. 
The equation (1.2) becomes, for the image functions, determined by the bilateral Laplace Carson transform,

$$
\left(p^{2} M+p C+K\right) u^{*}(p)=f^{*}(p)
$$

The sum $p \boldsymbol{C}+\boldsymbol{K}$ is derived from the constitutive laws of the members of the dynamic system dealt with. In case of satisfying a Kelvin - Voigt constitutve law, this sum can be rewritten as

$$
\boldsymbol{K}_{K V}^{\#}(p)=p \boldsymbol{C}+\boldsymbol{K}
$$

where the subscripts mean the Kelvin - Voigt law.

In the general case, when various constitutive laws may work, the equation characterizing the eigenvalue problem will be written as:

$$
\left[-\mu^{2} \boldsymbol{M}+\boldsymbol{K}^{\#}(p)\right] \boldsymbol{v}=0
$$

where $\boldsymbol{K}^{\#}(p)$ corresponds to specific constitutive laws characterizing the various members of the dynamic system dealt with.

\section{A critical look at the use of the Kelvin - Voigt consttutive law}

The need for a critical reconsideration of the equation (1.2) is determined by the fact that the constitutive laws implicitly postulated in the formulation of this equation are of Kelvin - Voigt type. This leads often to results that are not confirmed by physical experience, while an attempt at adopting a different type of constitutive laws would lead to considerable difficulties for the calculation techniques in case one tries to deal with the original functions.

A (simplistic) frequently encountered approach in structural dynamics is represented by postulating for the beginning the existence of ideally elastic constitutive laws, which leads for the equation (1.3) to a classical eigenvalue problem, for which the non-trivial solution is represented by a system of constant and real eigenvalues $\mu_{r}$ and eigenvectors $\boldsymbol{v}_{r}$. Thereafter, a correction is introduced just for the eigenvalues, in a way that is similar to the one which is rigorously usable only in case of dealing with single degree of freedom systems. The adoption of this simplistic approach keeps the eigenvectors real, while the eigenvalues become complex functions (with positive values of the imaginary parts) of the variable $p$.

A more correct procedure would lead to the need to consider together all three matrices occurring in the equations (1.4). It is shown [1], [5], that the eigenvalue problem derived,

$$
\left(-\mu^{2} \boldsymbol{M}+\mathrm{i} \mu \boldsymbol{C}+\boldsymbol{K}\right) \boldsymbol{v}=0
$$

is in general irreducible to a classical eigenvalue problem for the equation:

$$
\left(-\mu^{2} \boldsymbol{M}+\boldsymbol{K}\right) \boldsymbol{v}=0
$$

It is reducible to that type of equation only in case the matrix $C$ can be represented as a linear combination of terms $\left[\boldsymbol{K}\left(\boldsymbol{M}^{(-1)} \boldsymbol{K}\right)^{j}\right]$, where the values of $j$ are integer, arbitrary. In case the eigenvalue problem corresponding to that equation is no longer reducible to a classical problem, a correct way, dealt with in literature, is as follows: the non-linear $n$ - dimensional problem (with respect to $\mu$ ) corresponding to the equation (1.3'), is replaced by a linear, $2 n$-dimensional one. In this latter case the matrices used become usually non-symmetrical, while the solutions become complex. Note that this latter approach is usable only in case Kelvin - Voigt constitutive laws are admitted.

Two ways are thus referred to in order to deal with the equation (2.4):

a. replacing the non-linear (matrix) equation, formulated for the case of the original $n-$ dimensional space, by means of a linear equation formulated for the case of a $2 n-$ dimensional space; 
b. using the Laplace - Carson transform (2.1) and dealing with a space of the variable $p$.

The way (a) [1], [5], [8], applied to the equation (1.4), introduces initially an auxiliary vector of dimension $n$, which, added to the vector $v$, builds a vector of dimension $2 n$. A $(2 n \times 2 n)$ matrix that has no longer symmetry built in. The $(2 n \times 2 n)$ matrix equation leads in general to complex eigenvectors and eigenvalues. The complex eigenvectors will lead to interaction of the normal modes in case of free vibrations.

Advantages of way (a): this way requires analyzing a single eigenvalue problem (having a dimension $2 n \times 2 n)$.

Disadvantage of way (a): its use is limited to the analysis exclusively of cases where the constitutive laws are of Kelvin - Voigt type.

The way (b), [7], [8] is dealt with in the subsequent subsections of the paper. Nevertheless, one can already anticipate some main features of the outcome.

Advantages of way (b): this way is usable for the analysis of eigenvalue problems corresponding to various possible constitutive laws.

Disadvantage of way (b): this way leads to solutions corresponding to a single value of the variable $b$. A parametric analysis (with respect to $p$ ) must be dealt with. Nevertheless, in case one establishes a network of points $p$, solving the corresponding eigenvalue problems might benefit from the proximity of different points of the $\{p\}$ space, by using some adapted program.

\section{Analytical developments}

\subsection{General}

A different approach [7], [8], is adopted in the paper. An image equation (2.2), where the case of a Kelvin - Voigt constitutive law is admitted, is referred to. The matrix $\boldsymbol{K}^{\#}(p)$ would become in that case equal to the matrix $p \boldsymbol{C}+\boldsymbol{K}$ of equation (2.3), dealt with previously. In order to set up the constitutive laws of structural components, the use of generalized Maxwell laws [6] is proposed [7], [8]. The generalized Maxwell law is as follows (see Annex I): an ideally elastic (Hooke) component is connected in parallel with several Maxwell type components. The solution adopted in this way benefits from two main advantages: on one hand, experimentally determined characteristics can be approximated upon a desired interval of the $\omega$-axis; on the other hand, there exist methodological advantages raised by the analytical properties of the laws postulated, characterized by the existence of poles (the matrix $K^{\#}(p)$ is, under these assumptions, meromorphic).

\subsection{Relations of structural dynamics for structures with various constitutive laws}

Returning to the equations (2.2), a system of real eigenvectors to simultaneously diagonalize the matrix triad $(\boldsymbol{M}, \boldsymbol{C}, \boldsymbol{K})$ of the equation of motion does not exist in the general case for systems having Kelvin - Voigt constitutive laws. The consequence of this fact is that, in the general case when the eigenvectors become complex, a transfer of energy between the free vibrations corresponding to different eigenmodes occurs. More generally, for a pair of matrices $\left[\boldsymbol{M}, \boldsymbol{K}^{\#}(p)\right]$, where the second matrix is variable, there does not exist a system of constant, real eigenvectors in case the analysis is performed for bilateral Laplace - Carson transforms (where $p$ is the complex, independent, variable, replacing the time argument $t$, specific to analysis in the field of original functions).

This situation has important consequences, considered subsequently. The property of orthogonality of eigenvectors corresponding to different eigenmodes is no longer satisfied. It is consequently appropriate to introduce some new concepts, namely the concepts of pseudoorthogonality and of pseudo-normalization, which generalize the classical concepts of orthogonality and normalization. 
Given the advantages of use of the solutions of eigenvalue problems derived for the equation of motion, which are illustrated in literature for various cases, a generalization to the non-classical case was looked for. Two orientations may be distinguished in these studies:

a) approaches which are aiming at the direct determination of singularities (more precisely, the zeroes of the determinant for the image (force / displacement) impedance matrix $\boldsymbol{Z}^{\# \wedge}(p)$ [1], [5]);

$$
Z^{\# \wedge}(p)=p 2 M+K^{\# \wedge}(p)
$$

these approaches are usable, practically, in case of adoption of Kelvin - Voigt constitutive laws;

b) approaches aimed at deriving the inverse matrix $Z^{(-1) \#}(p)$ of the matrix $Z^{\#}(p)$, which is a function of the $p$ variable [7]; this way is the only one dealt with subsequently, due to its more general usability.

Following developments are starting from the equation (2.4), where both matrices $\boldsymbol{M}$ and $\boldsymbol{K}^{\#}(p)$ are symmetrical and lead to the eigenvalues $\lambda_{r}^{\#}(p)$ depending on the $p$ parameter, for the homogeneous equation

$$
\left[-\lambda^{\#}(p) \boldsymbol{M}+\boldsymbol{K}^{\#}(p)\right] \boldsymbol{v}^{*}(p)=0
$$

for which the non-trivial solutions (in case the value of variable $p$ to which the solution refers is not affected by singularities), is represented by the eigenvalues $\lambda_{r}^{\#}(p)$ and the corresponding eigenvectors $\boldsymbol{v}_{\boldsymbol{r}}{ }^{*}(p)$. The existence of non-trivial solutions implies for the equation zeroes for the determinant of $\left[-\lambda^{\#}(p) \boldsymbol{M}+\boldsymbol{K}^{\#}(p)\right]$,

$$
\text { Det }\left\{-\lambda^{\#} \boldsymbol{M}+\boldsymbol{K}^{\#}(p)\right\}=0
$$

\subsection{The case of points $p$ where the eigenvalues are different (i. e. simple)}

Due to the symmetry properties of matrices, in case of two different eigenvalues $\lambda_{r}^{\#}(p)$ and $\lambda_{s}^{\#}(p)$, the corresponding eigenvectors $\boldsymbol{v}_{r}^{\#}(p)$ and $\boldsymbol{v}_{s}^{\#}(p)$ are pseudo-orthogonal with respect to both matrices:

$$
\begin{array}{ll}
\boldsymbol{v}_{r}^{\#}(p)^{\mathrm{T}} \boldsymbol{M} \boldsymbol{v}_{s}^{\#}(p)=0 & (r \neq s) \\
\boldsymbol{v}_{r}^{\#}(p)^{\mathrm{T}} \boldsymbol{K}^{\#}(p) \boldsymbol{v}_{s}^{\#}(p)=0 & (r \neq s)
\end{array}
$$

(note: orthogonality would have involved that one of the factor vectors should be replaced by its complex conjugate).

In a similar way, the pseudo-norm (with respect to matrix $\boldsymbol{M}$ ) of a vector $\boldsymbol{v}^{\#}(p), m^{\#}(\boldsymbol{v})$, is defined by the relation:

$$
m^{\#}(\boldsymbol{v})^{2}=\boldsymbol{v}^{\#}(p)^{\mathrm{T}} \boldsymbol{M} \boldsymbol{v}^{\#}(p)
$$

while the pseudo-normalized with respect to matrix $\boldsymbol{M}$ homologous vector $\boldsymbol{v}^{\prime}(p), \boldsymbol{v}^{\#(M)}(p)$, is given by the relation:

$$
\boldsymbol{v}^{\#(M)}(p)=\boldsymbol{v}^{\#}(p) / m^{\#}(\boldsymbol{v})
$$

In order to formulate some condensed relations, it is appropriate to define the matrix of eigenvectors, $\boldsymbol{V}^{\#}(p)$. Its columns are represented by the eigenvectors $\boldsymbol{v}_{r}^{\#}(p)$ (arranged in the order of eigenvalues of rank $(r)$ ). In the same way, it is possible to define a matrix of pseudo-normal eigenvectors, $\boldsymbol{V}^{\#(M)}(p)$. The condition of pseudo-normalization may be rewritten as:

$$
\left.\boldsymbol{v}_{r}^{\#(M) T}(p) \boldsymbol{M} \boldsymbol{v}_{r}^{\#(M)}(p)=\delta_{\mathrm{rs}} \quad\left(\delta_{\mathrm{rs}}: \text { Kronecker's symbol }\right)\right)
$$

while a homologous relation for the matrix $\boldsymbol{K}^{\#}(p)$ is:

$$
\mathbf{v}_{\mathrm{r}}^{\#(\mathrm{M}) \mathrm{T}}(\mathrm{p}) \mathbf{K}^{\#}(\mathrm{p}) \mathbf{v}_{\mathrm{s}}^{\#(\mathrm{M})}(\mathrm{p})=\left[\lambda_{\mathrm{r}}^{\#}(\mathrm{p}) \lambda_{\mathrm{r}}^{\#}(\mathrm{p})\right]^{1 / 2} \delta_{\mathrm{rs}}
$$

The vectors $\boldsymbol{v}_{r}{ }^{\#(M)}(p)$ span mono-dimensional subspaces which are invariant with respect to the pair of matrices $\left(\boldsymbol{M}, \boldsymbol{K}^{\#}(p)\right)$. 
The relations

$$
\begin{aligned}
& \boldsymbol{V}^{(M) \# T}(p) \boldsymbol{M} \boldsymbol{V}^{\#(M)}(p)=\boldsymbol{I}_{n} \quad\left(\boldsymbol{I}_{n} \text { : unit matrix of dimension } n\right) \\
& \boldsymbol{V}^{(M) \# T}(p) \boldsymbol{K}^{\#}(p) \boldsymbol{V}^{\#(M)}(p)=\operatorname{Diag}\left\{\lambda_{r}^{\#}(p)\right\} \\
& \boldsymbol{V}^{(M) \# T}(p) \boldsymbol{M} \boldsymbol{K}^{\#(-1)}(p) \boldsymbol{M} \boldsymbol{V}^{\#(M)}(p)=\operatorname{Diag}\left\{1 / \lambda_{r}^{\#}(p)\right\} \\
& \boldsymbol{V}^{(M) \# T}(p) \boldsymbol{Z}^{\#}(p)^{\#} \boldsymbol{V}^{\#(M)}(p)=\operatorname{Diag}\left\{p^{2}+\lambda_{r}^{\#}(p)\right\} \\
& \boldsymbol{V}^{(M) \# T}(p) \boldsymbol{M} \boldsymbol{Z}^{(-1) \#}(p) \boldsymbol{M} \boldsymbol{V}^{(M) \#}(p)=\operatorname{Diag}\left\{1 /\left[p^{2}+\lambda_{r}^{\#}(p)\right]\right\}
\end{aligned}
$$

(where the symbol Diag means a diagonal matrix of dimension $n$, the matrix $\boldsymbol{V}^{(M) \#}(p)$ consists of columns equal to the eigenvectors $\boldsymbol{v}_{s}^{\#(M)}(p)$, while the impedance matrix, $\boldsymbol{Z}^{\#}(p)$, is defined by the relation (4.7) are satisfied.

Conversely, the relations (having the sense of spectral expansions for the matrices of the dynamic system dealt with) are

$$
\begin{aligned}
& \boldsymbol{K}^{\#}(p)=\boldsymbol{M} \boldsymbol{V}^{(M) \#}(p) \operatorname{Diag}\left\{\lambda_{r}^{\#}(p)\right\} \boldsymbol{V}^{(M) \# T}(p) \boldsymbol{M} \\
& \boldsymbol{K}^{\#(-1)}(p)=\boldsymbol{V}^{\#(M)}(p) \text { Diag }\left\{1 / \lambda_{r}^{\#}(p)\right\} \boldsymbol{V}^{\#(M) T}(p) \\
& \boldsymbol{Z}^{\#}(p)=\boldsymbol{M} \boldsymbol{V}^{\#(M)}(p) \operatorname{Diag}\left\{p^{2}+\lambda_{r}^{\#}(p)\right\} \boldsymbol{V}^{\#(M) T}(p) \boldsymbol{M} \\
& \boldsymbol{Z}^{\#(-1)}(p)=\boldsymbol{V}^{\#(M)}(p) \operatorname{Diag}\left\{1 /\left[p^{2}+\lambda_{r}^{\#}(p)\right]\right\} \boldsymbol{V}^{\#(M) T}(p)
\end{aligned}
$$

It may be shown that the properties accepted for the matrix $\boldsymbol{K}^{\#}(p)$ lead to the conclusion that the real and imaginary parts of the eigenvalue $\lambda_{r}^{\#}(p)$ satisfy the conditions

$$
\begin{aligned}
& \operatorname{Re} \lambda_{r}^{\#}(p)>0 \text { for } \operatorname{Re} p \geq 0 \text { and } \\
& \operatorname{Im} \lambda_{r}^{\#}(p) / \operatorname{Im} p \geq 0 \text { for the whole plane }\{p\},
\end{aligned}
$$

while the poles of the eigenvalues $\lambda_{r}^{\#}(p)$ can be placed only along the half-axis $(\operatorname{Im} p=0, \operatorname{Re} p<$ 0 ) in case the scalar constitutive laws of types (I.2) presented in Annex I can be directly applied as constitutive laws between the specific vectors of internal forces and the specific deformation components. It may be shown also that the eigenvalues $\lambda_{r}^{\#}(p)$ are stationary at the point $\boldsymbol{v}=$ $\boldsymbol{v}_{r}^{\#}(p)$, in case one considers the kind of variation of the expression $\lambda_{r}^{\#}(p)=\boldsymbol{v}^{T}(p) \boldsymbol{K}^{\#}(p) \boldsymbol{v}^{\#}(p)$ along the hyper-pseudosphere $\boldsymbol{v}^{T} \boldsymbol{M} \boldsymbol{v}=1$.

The matrix $\boldsymbol{K}^{\#}(p)$ and the eigenvalues $\lambda_{r}^{\#}(p)$ may be expanded into integer series of powers of $\left(p-p_{0}\right)$ [4]. The eigenvectors $\boldsymbol{v}_{r}^{\#}(p)$ are uniform functions, which may be expanded in a similar way into series of powers. Due to the condition (2.3), and to the fact that a complete basis exists, the eigenvectors do not have, at such points $p$, zeroes or poles.

\subsection{The case of points $p=p$, where multiple eigenvalues exist}

The case of points $p=p^{\prime}$, where multiple eigenvalues $\lambda_{r}^{\#}(p)$ exist, raises special problems, which impose a special kind of analysis, needing a revision of the calculation techniques usually adopted for points $p$, where all eigenvalues are different. Some features of the variation of the eigenvectors in the neighborhood of points $p=p$, where a multiple eigenvalue $\lambda_{r}^{\#}$, $(p)$ exists, may be mentioned: the variation of the system of eigenvectors in the subspace spanned by the system of eigenvectors corresponding to a multiple eigenvalue $\lambda_{r}^{\#},(p)$ must be replaced by a chain of principal vectors [10]. The diagonal submatrix corresponding to the chain referred to will no longer be a diagonal one, but is to be replaced by a submatrix of Jordan's canonic type [10]. The relations developed previously in subsection 4.3 of the paper must be correspondingly adapted. As mentioned in the note of subsection 2.1, item 1, in the problems dealt with in the paper this is not expected to happen. 


\section{Illustrative application}

The physical problem dealt with is related to the examination of the vibration of a dynamic system $S$ consisting of a mass connected by means of a perfectly elastic spring to a (vertical) axis-symmetrical foundation block that is connected at its turn to the elastic half-space. Note here that the connection to the half-space involves dissipative properties even in case of an ideally elastic half-space. This is due to the fact that during the vibration process the energy is radiated from the foundation block to the half-spacc, without returning to the contact zone. In order to keep calculations as simple as possible, the dissipative properties of the contact zone are (arbitrarily) assumed to correspond to a Kelvin - Voigt constitutive model. In agreement with the modelling and the approximate relations given in [2], the contact of an axis-symmetrical block with the half-space is equivalent to a dynamic single degree of freedom system, for which following input data were adopted:

- the mass of the rigid foundation block, including the equivalent mass pertaining to the half space material: $m_{l}=15 \mathrm{t}$;

- the viscous stiffness of the system of contact with the half-space: $c_{1}=4500 \mathrm{t} / \mathrm{s}=4500 \mathrm{kNs} / \mathrm{m}$;

- the elastic stiffness of the same contact system: $k_{l_{-}}=3000000 \mathrm{t} / \mathrm{s}^{2}=3000000 \mathrm{kN} / \mathrm{m}$;

- the mass of the upper body: $5 \mathrm{t}$;

- the viscous stiffness of the contact system between the two bodies: $c_{2}=0$;

- the elastic stiffness of the same: $k_{2}=100000 \mathrm{t} / \mathrm{s}^{2}=100000 \mathrm{kN} / \mathrm{m}$

The condition of zero value of the determinant corresponding to the equation of motion is

$$
m_{1} m_{2} \lambda^{\# 2}-\left[m_{1} k_{2}+m_{2}\left(c p+k_{1}+k_{2}\right)\right] \lambda^{\#}+k_{1} k_{2}=0
$$

with the solutions for the eigenvalues

$$
\begin{aligned}
& \lambda_{I, I I}^{\#}(p)=<\left[m_{1} k_{2}+m_{2}\left(c p+k_{1}+k_{2}\right)\right]-/+ \\
& -/+\left\{\left[m_{1} k_{2}+m_{2}\left(c p+k_{1}+k_{2}\right)\right]^{2}-4 m_{1} m_{2} k_{1} k_{2}\right\}^{1 / 2}>/\left(2 m_{1} m_{2}\right)
\end{aligned}
$$

and for the eigenvectors respectively

$$
\begin{aligned}
& v_{1 r}{ }^{\#}=\left(k_{2}-\lambda_{r}^{\#} m_{2}\right) / n_{r}{ }^{\#} \\
& v_{2 r}{ }^{\#}=k_{2} / n_{r}{ }^{\#}
\end{aligned}
$$

where the denominator $n_{r}^{\#}$ has the expression

$$
n_{r}^{\#}=\left[m_{1}\left(k_{2}-\lambda_{r}^{\#} m_{2}\right)^{2}+m_{2} k_{2}^{2}\right]^{1 / 2}
$$

The solutions (eigenvalues and eigenvectors) as functions of the non-dimensional parameter $c \omega /$ $k_{l}$, assuming $p=\mathrm{i} \omega$ (all of them complex), are presented in following figures numbered 1-6 respectively for the non-dimensional interval $(0,2.0)$, of the argument $c \omega / k_{1}$. The solutions $\lambda_{r}^{\#}$ are presented in $2 \times 1$ plots, while the solutions $v_{I}^{\#}$, are presented in $2 \times 2$ plots.

To note that figures a are used for colors blue (for the real parts) and figures $b$ are used for colors red (for the imaginary parts). It may be remarked that a singularity occurs for the eigenvector $\boldsymbol{v}_{I,}^{\#}$ at a value of about 0.15 of the non-dimensional ratio $c \omega / k_{l}$. Examining the plots presented, it turns out that for the system dealt with a strong dependence on the non-dimensional argument exists. Of course, one must take into account the fact that the results presented concern directly the Laplace - Carson images and that a use of them for practical purposes involves in principle a conversion to the field of originals for the functions of interest. 
Lamda

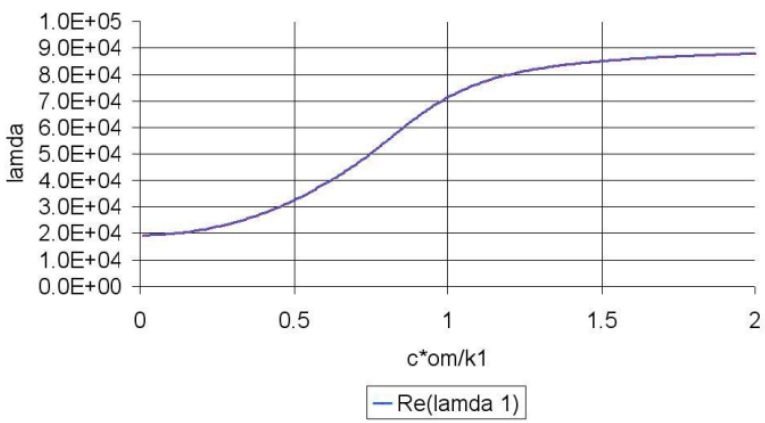

Fig. 1.a. - Real part for $\lambda_{I}^{\#}$

Lamda

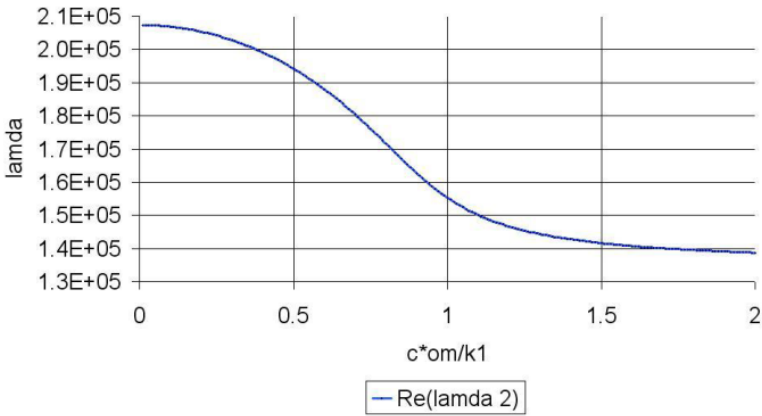

Fig. 2.a. - Real part for $\lambda_{I I}^{\#}$

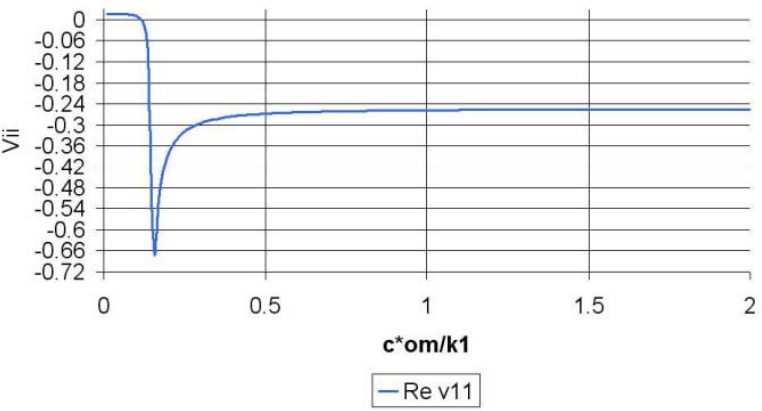

Fig. 3.a. - Real part of component $v_{l r}^{\#}$

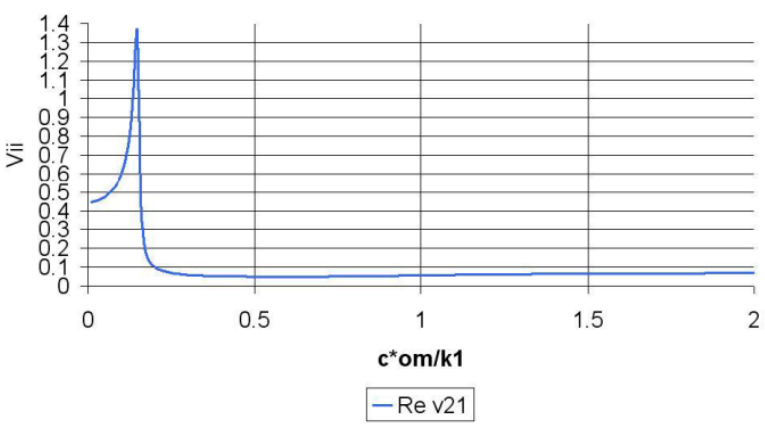

Fig. 4.a. - Real part of component $v_{2 r}^{\#}$

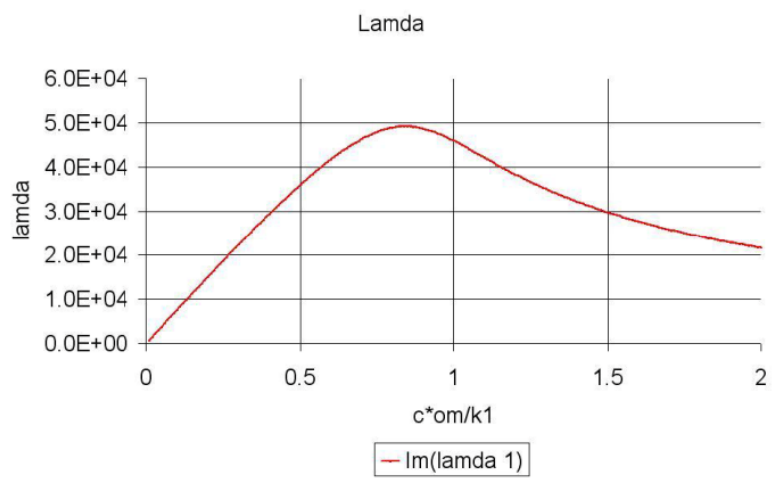

Fig. 1.b. - Imaginary part for $\lambda_{I}^{\#}$

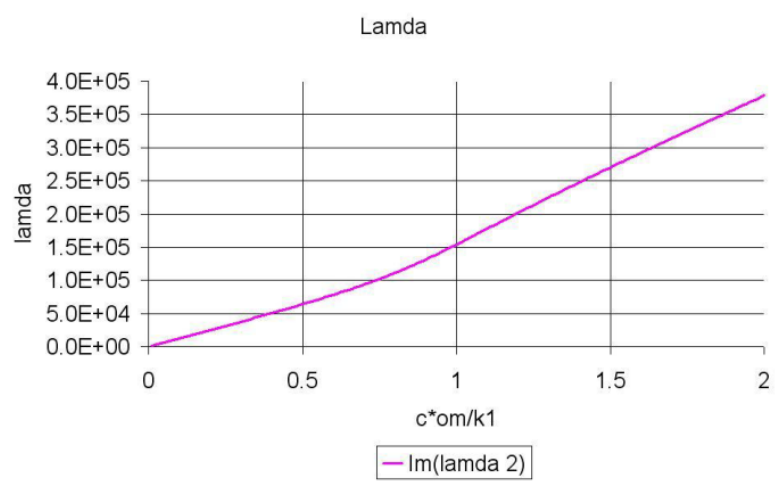

Fig. 2.b. - Imaginary part for $\lambda_{I I}^{\#}$

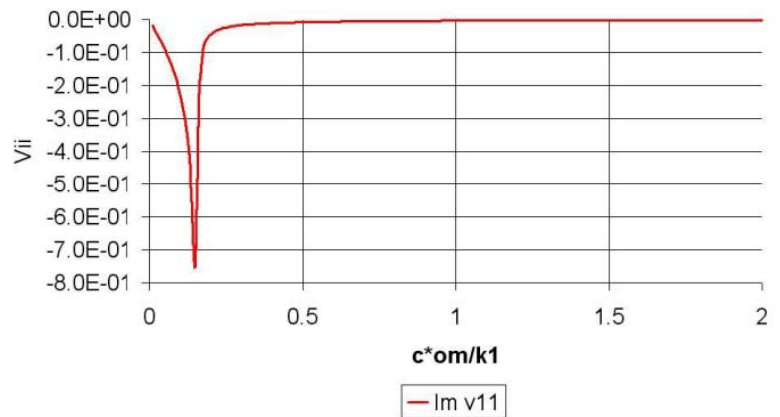

Fig. 3.b. - Imaginary part of component $v_{l r}^{\#}$

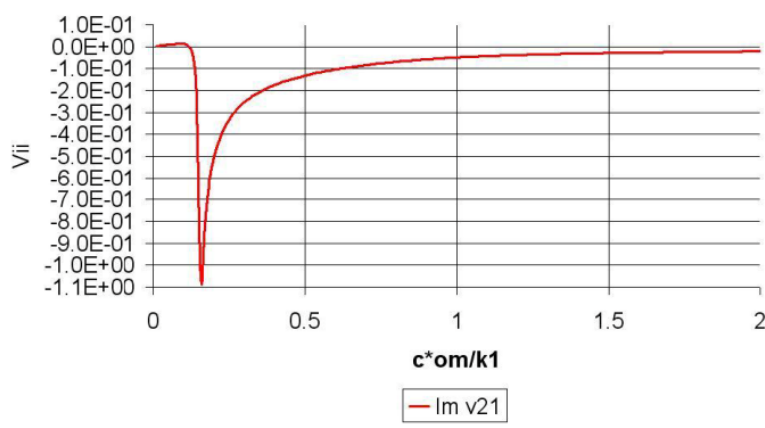

Fig. 4.b. - Imaginary part of component $v_{2 r}{ }^{\#}$ 


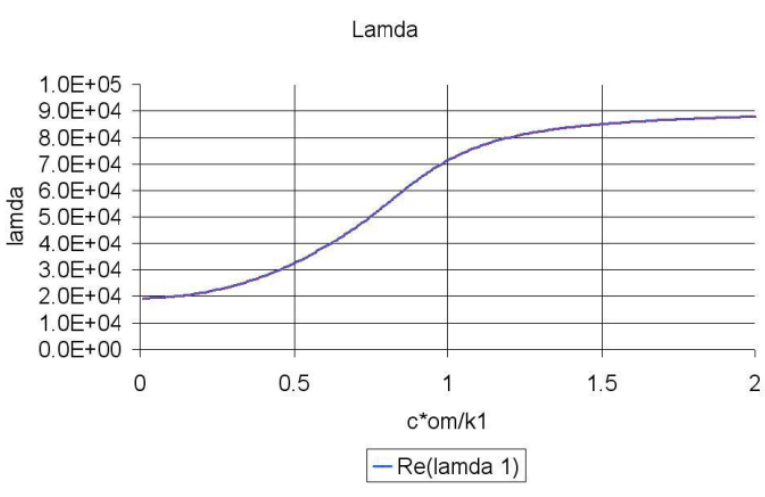

Fig. 5.a. - Real part of components of eigenvector $\boldsymbol{v}_{I}^{(M)}{ }_{I}^{\#}(p)$

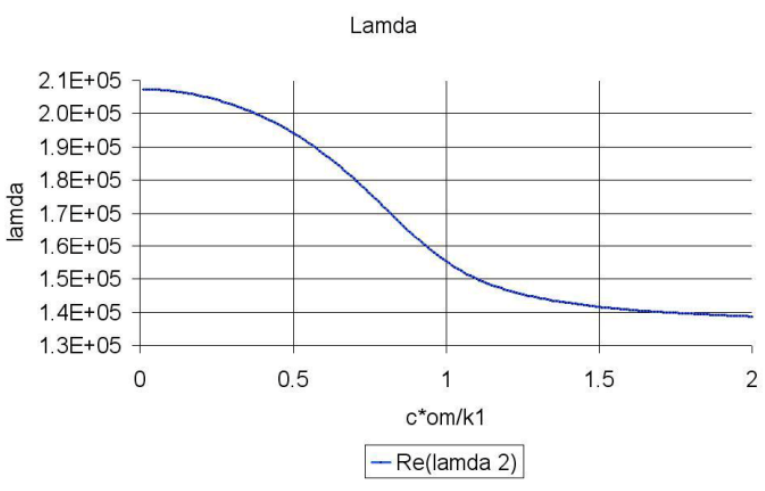

Fig. 6.a. - Real part of components of eigenvector $\boldsymbol{v}^{(M)}{ }_{I I}^{\#}(p)$

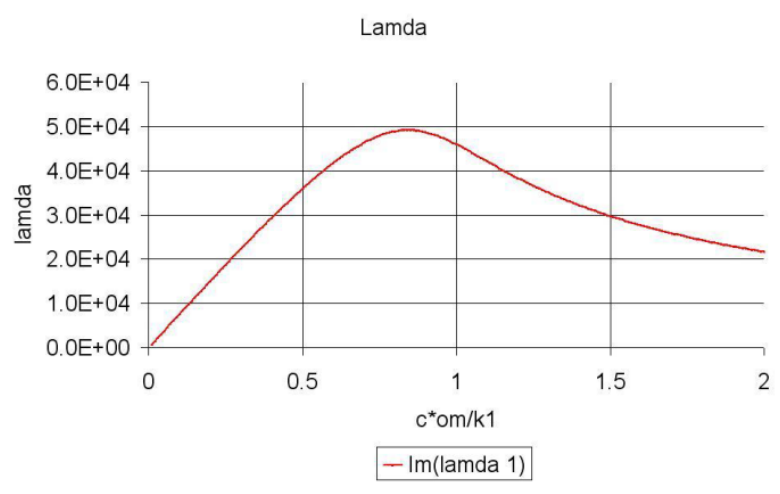

Fig. 5.b. - Imaginary part of components of eigenvector $\boldsymbol{v}_{I}^{(M)}{ }_{I}^{\#}(p)$

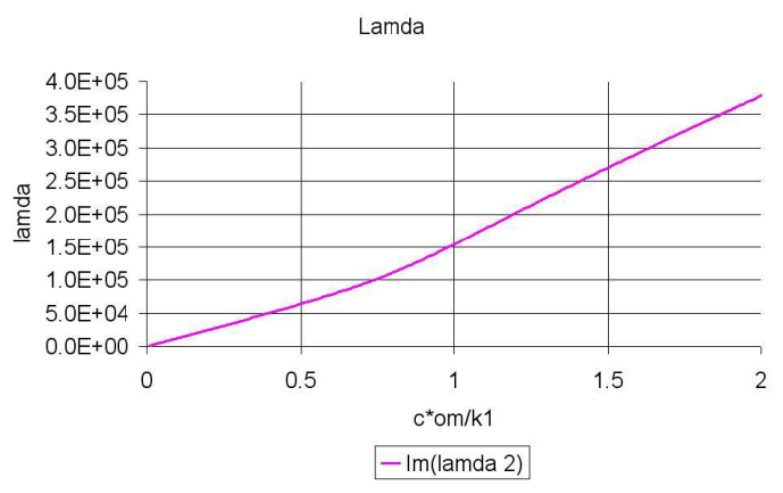

Fig. 6.b. - Imaginary part of components of eigenvector $\boldsymbol{v}_{I I}^{(M)}(p)$

\section{Final considerations}

The paper presented is dealing with a problem of obvious interest, namely that of contributing to the adoption of an instrument of analysis of the performance of dynamic systems having components characterized by linear constitutive laws of a quite high complexity. This may lead to analyses to be more realistic than the practically exclusive use of Kelvin - Voigt constitutive laws, which are so frequently encountered in the literature, without the required comments.

The use of the bilateral Laplace - Carson transform represents a highly efficient tool of analysis. Becoming familiar with this procedure is recommended to those engaged in the linear analyses of various problems of structural dynamics.

Besides the direct transform, expressed by the relation (2.1a), which consists of usual integration, the use of the inverse transform $(2.1 \mathrm{~b})$ based on the residue theorem of the theory of complex functions, is highly recommendable.

The appropriate consideration of the convergence band $(\alpha, \beta)$ that is specific to the various functions dealt with should be carefully carried out.

While the rather traditional procedure of dealing with dynamic systems characterized by Kelvin - Voigt constitutive laws (2.3), which consists to a passing from a nonlinear problem for an $n$ dimensional to a linear problem for a $2 n$-formulation leads to a unique solution, the alternative way presented in the paper will deal with a family of $n$-dmensional formulations depending upon the complex variable $p$. This means, of course, a huge increase of the computing volume, but it seems to be feasible in case appropriate algorthms are developed.

Studies on deriving most appropriate expressions of constitutive laws are of course desirable. 


\section{References}

[1] Caughey T. K., O'Kelly M. E. J.(1963), General theory of vibration of damped linear dynamic systems, California Institute of Technology.

[2] Clough R., Penzien J. (1975). Dynamics of structures. Prentice Hall, Englewood Cliffs.

[3] Faddeev D. K., Faddeeva V. N. (1960). Vychisliltelnye metodî lineinoy alghebrî. GIFML, Moscow.

[4] Knopp K.(1930). Funktionentheorie. Goeschen, Heidelberg.

[5] O'Kelly M. E. J. (1964). Vibration of viscously damped linear dynamic systems. Thesis, California Institute of Technology.

[6] Reiner M. (1964). Reologhia, GIFML, Moscow.

[7] Sandi H. (1970). Eigenwertaufgaben und Übertragungsmatrizen für nichtkonservative mechanische Systeme. ZAMM, 5.

[8] Sandi H. (1983). Elemente de dinamica structurilor. Editura Tehnică, Bucuresti.

[9] Van der Pol. B., Bremmer H. (1950). Operational calculus based on the two - sided Laplace transform. Oxford.

[10] Wilkinson J. H. (1965). The algebraic eigenvalue problem. Oxford Univ. Press.

\section{Annex 1. Properties of some constitutive laws}

To start, a discussion on some alternative constitutive laws is dealt with. These laws should allow defining the most appropriate kind of equations of motion for the dynamic systems investigated. Keeping in view the fact that the main tool for analysis is represented by the use of the bilateral Laplace - Carson transform (2.1), this approach is based on the use of the corresponding transforms of original functions depending on time. Two basic entities are considered, for illustration of the use of the transforms referred to: the normal stress, $\sigma^{\sharp}(p)$ and the homologous local deformation $\varepsilon^{\sharp}(p)$. An explicite extension to tensorial functions is not necessary at this place in this respect.

Two reference models, in which the elasticity (or elastic stiffness) modulus, $E$, and the viscous stiffness modulus, $\eta$, intervene, are used [6]:

- the ideally elastic model (called Hooke's model), $\quad \sigma^{\#}(p)=E \varepsilon^{\#}(p)$

- $\quad$ the ideally viscous model (called Newton's model), $\sigma^{\sharp}(p)=p \eta \varepsilon^{\#}(p)$

These models are to be dealt with in adequate ways for performing specific analyses. The parameters $E$ and $\eta$ are first used for connections in parallel or in series respectively and are to be combined in an appropriate way to correspond to various goals of analysis.

The model of solid body with retardation (called the Kelvin - Voigt model):

$$
\begin{aligned}
& \sigma^{\#}(\mathrm{p})=\mathrm{E} \varepsilon^{\#}(\mathrm{p})+\mathrm{p} \eta_{\text {ret }} \varepsilon^{\#}(\mathrm{p})=\left(\mathrm{E}+\mathrm{p}_{\text {ret }} \eta\right) \varepsilon^{\#}(\mathrm{p})= \\
& =\mathrm{E}\left(1+\mathrm{p} \eta_{\text {ret }} / \mathrm{E}\right) \varepsilon^{\#}(\mathrm{p})=\mathrm{E}\left(1+\mathrm{p} \mathrm{T}_{\text {ret }}\right) \varepsilon^{\#}(\mathrm{p})
\end{aligned}
$$

The model of viscous fluid body with relaxation (called the Maxwell model):

$$
\begin{aligned}
& \varepsilon^{\#}(p)=\sigma^{\sharp}(p) / E+p \sigma^{\sharp}(p) / \eta_{r e l}, \\
& \sigma^{\#}(p)=\left[p E \eta_{\text {rel }} /\left(E+p \eta_{r e l}\right)\right] \varepsilon^{\sharp}(p)=\left[E /\left(1+p \mathrm{~T}_{\text {rel }}\right)\right] \varepsilon^{\#}(p)
\end{aligned}
$$

The two latter models include two parameters of physical dimension time: the retardation time, $\mathrm{T}_{\mathrm{ret}}$, and the relaxation time, $\mathrm{T}_{\mathrm{rel}}$, respectively.

A first combination (in parallel) of these two models is the Poynting model

$$
\sigma^{\#}(p)=\left[E_{0}+E_{1} /\left(1+p \mathrm{~T}_{\mathrm{ret}}\right)\right] \varepsilon^{\#}(p)
$$

while a generalization of it is the generalized Maxwell model,

$$
\sigma^{\#}(p)=\left[E_{0}+\Sigma_{k} E_{k} /\left(1+p \mathrm{~T}_{\text {ret.k }}\right)\right] \varepsilon^{\#}(p)
$$

The scalar models (I.1) and (I.2) may be extended to pluri - dimensional models, in order to derive specific laws to structural models. The use of the model corresponding to relation (I.2d) is subsequently preferred. This is because such an option makes it possible to approximate the rheological properties of materials upon a convenient frequency band and, at the same time, offer controllable singularities (poles of the theory of functions of a complex variable). 
NOTE:

The fact that the denominators of the terms of expressions (I.2d), as well as of the denominator of expression (I.2c), have real, positive, values leads to the fact that the poles intervening in the expressions of terms of index $k$ are placed on the negative half-axis, $\operatorname{Re} p<0, \operatorname{Im} p=0$, at abscissae of $\left(-1 / \mathrm{T}_{\text {ret.k. }}\right)$.

The coefficients $E_{k}$ of expression (I.2d) have the same physical dimension as that of the coefficient $E_{0}$, while their physical sense is to be specified for each of them.

\section{Annex II. Equations and solutions in cases of deterministic disturbances}

The following displacement vectors concerning the motion are used for the subsequent developments:

- the system of displacements along the degrees of freedom of the ground - structure interface, denoted $\boldsymbol{u}^{* i n t}(p)$ (degrees of freedom: $i=1 \ldots m$ ),

- the system of displacements along the degrees of freedom of the part of structure in elevation (which do not include the previous ones), denoted $\boldsymbol{u}^{* \text { ele }}(p)$ (degrees of freedom: $j=1 \ldots n)$

A first basic case considered, $C .1$, is represented by the existence of given data concerning the vector of image forces $f^{* \text { ele }}(p)$, which defines in this case the input applied to the dynamic system in elevation dealt with. The equation (1.4) is rewritten as

$$
Z^{\#}(p) \boldsymbol{u}^{* \text { ele }}(p)=\boldsymbol{f}^{* \text { ele }}(p)
$$

and its solution $\boldsymbol{u}^{* e l e}(p)$, which defines the output, is

$$
\boldsymbol{u}^{*^{e l e}}(p)=Z^{\#}(p)^{\#(-1)} \boldsymbol{f}^{*^{e l e}}(p)=\boldsymbol{V}^{(M) \#}(p) \operatorname{Diag}\left\{1 /\left[p^{2}+\lambda_{r}^{\#}(p)\right]\right\} \boldsymbol{V}^{(M) \# T}(p) \boldsymbol{f}^{*^{e l e}}(p)
$$

Another basic case of interest, C.2, is that, when a structure supported by the "ground" is subjected to the ground - structure interface motion, representing the input data. In this case it becomes necessary to define two different categories of displacements, pertaining to the degrees of freedom characterizing the entities dealt with:

- the general case (input: $m$ - dimensional, $i=1 \ldots m$ ) concerns the system of degrees of freedom corresponding to the ground - structure interface, for which the corresponding displacement vectors, playing the role of input, are denoted $\boldsymbol{u}^{* i n t}(p)$;

- the other one ( $n$-dimensional, $j=1 \ldots n)$ concerns the degrees of freedom corresponding to the parts of the structure in elevation, for which the corresponding displacement vectors, playing the role of output, are denoted $\boldsymbol{u}^{* \text { ele }}(p)$.

A first subcase, C.2.1, is represented by the existence of arbitrary vectors $\boldsymbol{u}^{* \text { int }}(p)$, while a second subcase, $C .2 .2$, is represented by the existence of a rigid body system of displacements, $\boldsymbol{u}^{*^{r i b}}(p)$, characterizing the interface motion. In subcase $C .2 .1$ one must consider a rectangular ( $n$ rows $\times m$ columns) matrix $\boldsymbol{R}^{* \text { ele }}(p)$, which converts the input vector $\boldsymbol{u}^{*^{\text {int }}}(p)$ into forces $\boldsymbol{f}^{* \text { ele }}(p)$,

$$
f^{* \text { ele }}(p)=\boldsymbol{R}^{* \text { ele }}(p) \boldsymbol{u}^{* i n t}(p)
$$

The matrix $\boldsymbol{R}^{* \text { ele }}(p)$ has following sense: a term of it represents the response of the system along the degree of freedom $j$ of the system in elevation to an input equal to 1.0 acting along the degree of freedom $i$ of the interface.

In subcase C.2.2 one must consider a rectangular ( $n$ rows $\times m$ columns) constant matrix $\boldsymbol{G}$, which converts the rigid body motion of the interface into rigid body motion of the part in elevation,

$$
\boldsymbol{u}^{*^{\text {elerig }}}(p)=\boldsymbol{G} \boldsymbol{u}^{*^{\text {int.rig }}}(p)
$$


The absolute displacements along the degrees of freedom of the elevation part of the dynamic system, $\boldsymbol{u}^{\boldsymbol{*}^{e l e . a b s}}(p)$, can be expressed as a sum of rigid body displacements $\boldsymbol{u}^{\boldsymbol{*}^{e l e . r i g}}(p)$ and of relative displacements $\boldsymbol{u}^{* \text { ele.rel }}(p)$,

$$
\boldsymbol{u}^{* \text { ele.abs }}(p)=\boldsymbol{u}^{*^{\text {ele.rig }}}(p)+\boldsymbol{u}^{*^{\text {ele.rel }}}(p)
$$

The term $\boldsymbol{u}^{* \text { ele.rig }}(p)$ contributes to the kinetic energy only, while the term $\boldsymbol{u}^{* \text { ele.rel }}(p)$ contributes to both terms, $p^{2} \boldsymbol{M} \boldsymbol{u}^{* \text { ele.rel }}(p)$ and $\left.\boldsymbol{K}^{\#}(p) \boldsymbol{u}^{*^{\text {ele.rel }}}(p)\right]$ of the left member of the equation of motion,

$$
\left[p^{2} \boldsymbol{M}+\boldsymbol{K}^{\#}(p)\right] \boldsymbol{u}^{* \text { ele.rel }}(p)=-p^{2} \boldsymbol{M} \boldsymbol{u}^{* \text { el.elrig }}(p)=-p^{2} \boldsymbol{M} \boldsymbol{G} \boldsymbol{u}^{*^{\text {int.rig }}}(p)
$$

one has as starting points the kinematics relation

and

$$
\boldsymbol{u}^{* a b s}(p)=\boldsymbol{u}^{*^{r i g}}(p)+\boldsymbol{u}^{* r e l}(p)
$$

$$
\left[p^{2} \boldsymbol{M}+\boldsymbol{K}^{\#}(p)\right] \boldsymbol{u}^{* a b s}(p)=\boldsymbol{f}^{* a b s}(p)
$$

In this case, it is necessary to consider two separate spaces:

- the space ( $m$ - dimensional) which concerns the degrees of freedom of the interface with vectors $\boldsymbol{u}^{* i n t}(p)$;

- the other space ( $n$ - dimensional) which concerns the degrees of freedom of the structure elevation, with vectors $\boldsymbol{u}^{*^{\text {ele }}}(p)$.

For the latter space, concerning the degrees of freedom of the structure elevation, it is necessary to consider further on:

- absolute displacements $\boldsymbol{u}^{* a b s}(p)$

- $\quad$ rigid body displacements $\boldsymbol{u}^{* \text { rig }}(p)$ and

- relative displacements, $\boldsymbol{u}^{* \text { rel }}(p)$ (compatible with the interface displacements $\boldsymbol{u}^{* \text { int }}(p)$ if rigid body interface displacements $\boldsymbol{u}^{* i n t}(p)$ exist).

In case one is dealing directly with absolute displacements, a cross-connection matrix $\boldsymbol{G}^{\#}(p)$ will be introduced. Its terms have the following meaning: the term $g_{j i}^{\#}(p)$ (where $j=1 \ldots n$ and $i=1 \ldots m)$ represents the component of action applied along the $j^{\text {th }}$ DOF of the $n$-dimensional space of actions in case the dynamic system is loaded exclusively by a displacement $u_{g i}=1$ applied to the $i^{\text {th }} m$-dimensional space of the interface between ground and structure.

Another case to be considered is represented by that of given rigid body motion of the ground structure interface. A frequently used way to deal with this case is represented by the introduction of relative displacements $\boldsymbol{u}^{* \text { rel }}(p)$. The rigid body displacements of elevation, $\boldsymbol{u}^{* \text { rig }}$ $(p)$, will be compatible with the rigid body displacements of the interface, $\boldsymbol{u}^{* \text { int }}(p)$. 